\title{
An ancient type of MnmA protein is an iron-sulfur cluster-dependent sulfurtransferase for tRNA anticodons
}

\author{
NAOKI SHIGI, ${ }^{1}$ MASAKI HORITANI,, ${ }^{2}$ KENJYO MIYAUCHI, ${ }^{3}$ TSUTOMU SUZUKI, $^{3}$ and MISAO KUROKI ${ }^{1}$ \\ ${ }^{1}$ Biotechnology Research Institute for Drug Discovery, National Institute of Advanced Industrial Science and Technology (AIST), 2-4-7 Aomi, \\ Koto-ku, Tokyo 135-0064, Japan \\ ${ }^{2}$ Faculty of Agriculture, Department of Applied Biochemistry and Food Science, Saga University, 1 Honjo-machi, Saga 840-8502, Japan \\ ${ }^{3}$ Department of Chemistry and Biotechnology, Graduate School of Engineering, University of Tokyo, 7-3-1 Hongo, Bunkyo-ku, \\ Tokyo 113-8656, Japan
}

\begin{abstract}
Transfer RNA (tRNA) is an adaptor molecule indispensable for assigning amino acids to codons on mRNA during protein synthesis. 2-thiouridine $\left(s^{2} U\right)$ derivatives in the anticodons (position 34 ) of tRNAs for glutamate, glutamine, and lysine are post-transcriptional modifications essential for precise and efficient codon recognition in all organisms. $s^{2} U 34$ is introduced either by (i) bacterial MnmA/eukaryote mitochondrial Mtu1 or (ii) eukaryote cytosolic Ncs6/archaeal NcsA, and the latter enzymes possess iron-sulfur ( $\mathrm{Fe}-\mathrm{S}$ ) cluster. Here, we report the identification of novel-type MnmA homologs containing three conserved Cys residues, which could support Fe-S cluster binding and catalysis, in a broad range of bacteria, including thermophiles, Cyanobacteria, Mycobacteria, Actinomyces, Clostridium, and Helicobacter. Using EPR spectroscopy, we revealed that Thermus thermophilus MnmA (TtMnmA) contains an oxygen-sensitive [4Fe-4S]-type cluster. Efficient in vitro formation of $s^{2} U 34$ in tRNA ${ }^{\text {Lys }}$ and tRNA ${ }^{\text {Gln }}$ by holo-TtMnmA occurred only under anaerobic conditions. Mutational analysis of TtMnmA suggested that the Fe-S cluster is coordinated by the three conserved Cys residues (Cys105, Cys108, and Cys200), and is essential for its activity. Evolutionary scenarios for the sulfurtransferases, including the Fe-S cluster containing $\mathrm{Ncs} 6 / \mathrm{NcsA} \mathrm{s}^{2} \mathrm{U}$ thiouridylases and several distantly related sulfurtransferases, are proposed.
\end{abstract}

Keywords: biosynthesis; iron-sulfur cluster; post-transcriptional modification; sulfurtransferase; tRNA

\section{INTRODUCTION}

During translation of genomic information into proteins, tRNAs deliver amino acids to their respective mRNA codons. There are numerous post-transcriptional chemical modifications in tRNA that are important for precise codon recognition and stabilization of the tRNA tertiary structure (Duechler et al. 2016; Boccaletto et al. 2018; Hori et al. 2018). Position 34 (the anticodon wobble base) of glutamate, glutamine, and lysine tRNAs is universally modified to 5-methyl-2-thiouridine derivatives $\left(\mathrm{xm}^{5} \mathrm{~s}^{2} \mathrm{U}\right)$ (Fig. 1A; Armengod et al. 2014; Schaffrath and Leidel 2017). These amino acids are encoded by two degenerate codons ending in purine in the two-codon boxes that specify two amino acids by the difference in the third codon bases. Because of the steric hindrance between the bulky 2-thiocarbonyl group and the $2^{\prime}$-hydroxyl group of $s^{2} U, x m^{5} s^{2} U$ preferentially adopts the C3'-endo form of ribose puckering (Yokoyama et al. 1985; Agris et al. 1992). $x^{5}{ }^{5} s^{2} U 34$ base-pairs more tightly with purines than with pyrimidines,

Corresponding author: naoki-shigi@aist.go.jp

Article is online at http://www.rnajournal.org/cgi/doi/10.1261/rna. 072066.119. are thus required for efficient codon selection (Ashraf et al. 1999; Vendeix et al. 2012; Rodriguez-Hernandez et al. 2013; Rozov et al. 2016; Ranjan and Rodnina 2017), and for preventing misreading of near cognate codons (Agris et al. 1973; Yokoyama et al. 1985; Johansson et al. 2008) and frame shifting (Urbonavicius et al. 2001). The absence of the 2-thio modification leads to ribosome stalling at Aending codons in mRNAs, and abnormal translation speed causes protein misfolding and aggregation (Nedialkova and Leidel 2015). In patients with myoclonus epilepsy with ragged-red fibers, the lack of the U34 modification in mutant mitochondrial tRNA ${ }^{\text {Lys }}$ induces abnormal translation leading to disease development (Yasukawa et al. 2001). The 2-thio modification in the $m^{5} s^{2} U\left(s^{2} T\right)$ at position 54 in the T-loop also plays a role in translation at high temperatures. In some thermophilic bacteria and archaea, nearly all of the tRNA species possess $m^{5} s^{2} U 54$

(C) 2020 Shigi et al. This article is distributed exclusively by the RNA Society for the first 12 months after the full-issue publication date (see http://rnajournal.cshlp.org/site/misc/terms.xhtml). After 12 months, it is available under a Creative Commons License (Attribution-NonCommercial 4.0 International), as described at http:// creativecommons.org/licenses/by-nc/4.0/. 
A

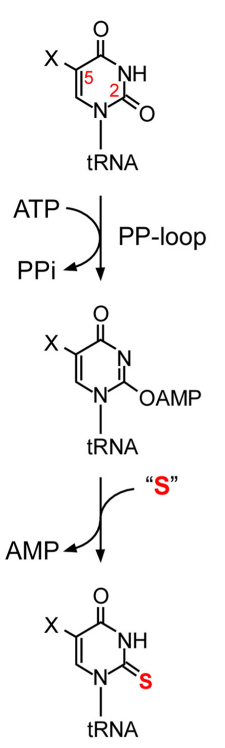

B
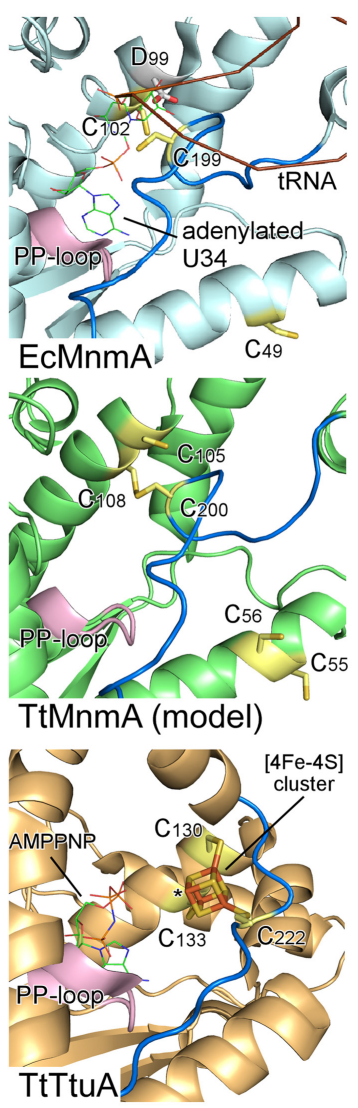

C

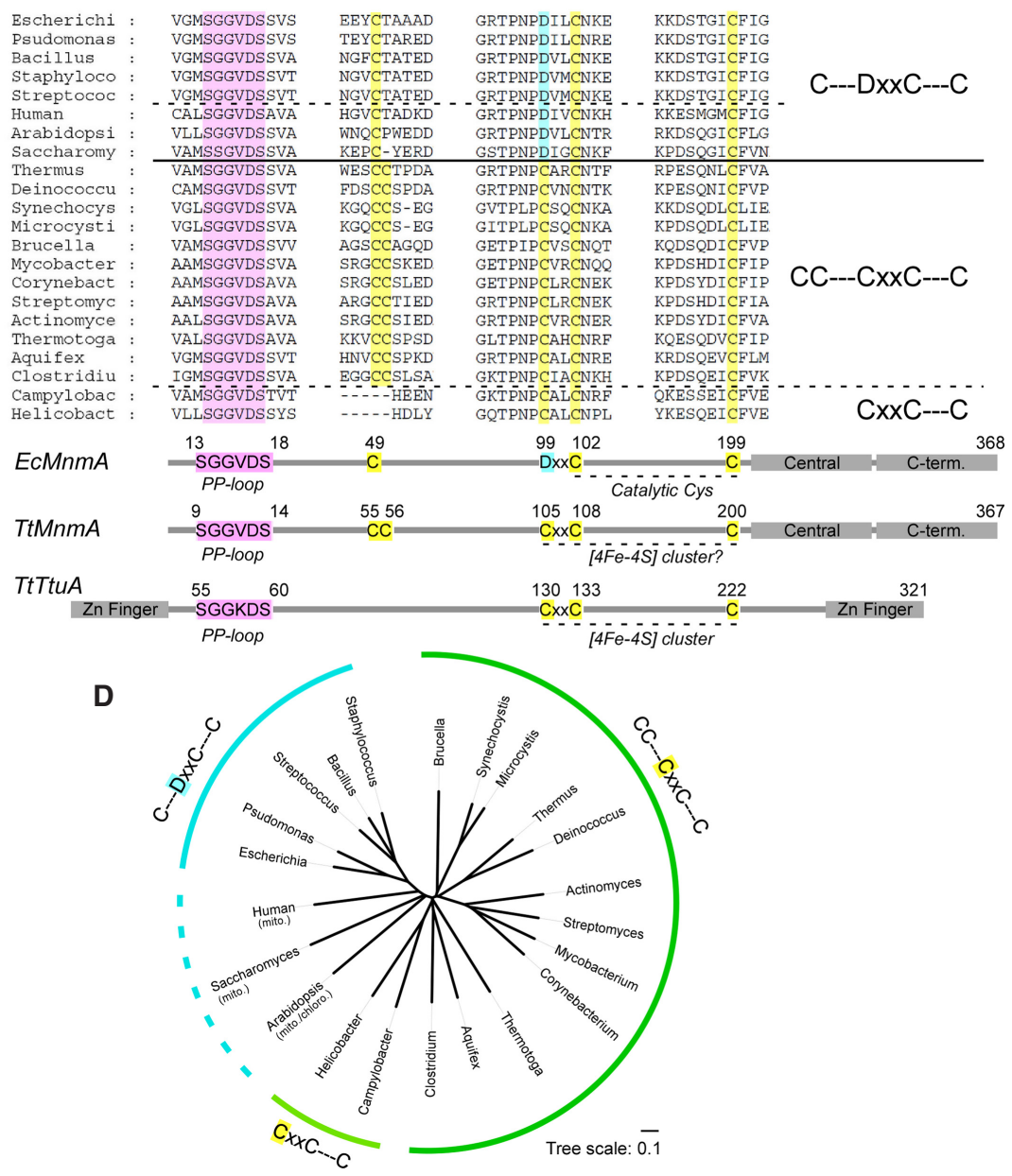

FIGURE 1. Two types of MnmA tRNA s² ${ }^{2} 34$ sulfurtransferases. (A) Reaction mechanism of MnmA. The PP-loop motif in MnmA is involved in the formation of an adenylated intermediate, and subsequent nucleophilic attack by activated sulfur (depicted as " $S^{\prime \prime}$ ) generates $s^{2} U$ and releases AMP. (B) Structures of the catalytic centers of representative RNA sulfurtransferases. Structures of EcMnmA (PDB: 2 deu), TtMnmA model generated by SWISS-MODEL, and TtTtuA (PDB: $5 \mathrm{~b} 4 \mathrm{e}$ ) were rendered by the PyMol program (DeLano Scientific). Important residues and ligands in these proteins are depicted and labeled. The Fe with a free coordination site in TtTtuA is labeled with an asterisk (*). The flexible loops with a cysteine residue are colored blue. $(C)$ Conserved sequence motifs in the catalytic domains of MnmAs from several representative bacteria and eukaryotes. These sequences were aligned with the CLUSTAL X2 program (Larkin et al. 2007) and visualized with the GeneDoc program. The complete alignment is shown in Supplemental Figure S1. Schematic representation of the conserved motifs in the catalytic domains of EcMnmA, TtMnmA, and TtTtuA is depicted below the alignment, along with other domains. (D) Phylogenic tree of several MnmAs drawn with the CLUSTAL X2 and TOL programs (Letunic and Bork 2016) based on the sequence alignment in C.

(Watanabe et al. 1974; Kowalak et al. 1994), in contrast to the conserved $\mathrm{m}^{5} \mathrm{U}(\mathrm{rT}) 54$ in mesophiles. 2-thiolation of $\mathrm{m}^{5} \mathrm{~s}^{2} \mathrm{U} 54$ is required for survival at high temperatures (Shigi et al. 2006a). $m^{5} s^{2} U 54$ base-pairs with $m^{1} A 58$ and stacks with $\mathrm{G} 53$ and $\Psi 55$. $\Psi 55$ and $\mathrm{C} 56$ base-pair with G18 and G19, respectively. The A-form helix formed by these interactions in the tRNA core is stabilized by the intrinsic rigidity of the $\mathrm{m}^{5} \mathrm{~s}^{2} \mathrm{U}$ structure (Watanabe et al. 1974; Horie et al. 1985).

The biosynthesis of tRNA sulfur modifications involves "sulfur trafficking systems" that begin with cysteine desulfurases and "modification enzymes" that directly incorporate sulfur into tRNAs (Shigi 2014, 2018). For $s^{2} U 34$ synthesis, two types of modification enzymes have been discovered: (i) bacterial MnmA (Hagervall et al. 1998; Kambampati and Lauhon 2003; Ikeuchi et al. 2006)/eukaryotic mitochondrial Mtu1 (Umeda et al. 2005) and (ii) eukaryotic cytosolic Ncs6 (Bjork et al. 2007; Dewez et al. 2008; Nakai et al. 2008; Leidel et al. 2009; Noma et al. 2009)/archaeal NcsA (Chavarria et al. 2014). For $s^{2}$ U54 synthesis, we identified TtuA, which is closely related to the Ncs6/NcsA family (Shigi et al. 2006a,b, 2008, 2016; Chen et al. 2017). These RNA sulfurtransferases contain a characteristic signature motif (SxGxDS/T, PP-loop), which binds to the pyrophosphate moiety of ATP (Bork and Koonin 1994). These PP-loop ATP pyrophosphatases first activate their substrates via adenylation and a subsequent nucleophilic attack by the activated sulfur (e.g., 
bisulfide, protein-persulfide, or protein-thiocarboxylate; depicted as "S") generates $s^{2} U$ (Fig. 1A; Ikeuchi et al. 2006; Shigi et al. 2008; Arragain et al. 2017; Chen et al. 2017). One sharp difference in these sulfurtransferases is the cofactor dependence: Only the Ncs6/NcsA/TtuA proteins require iron-sulfur (Fe-S) clusters for their activity. In this study, we found that MnmA homologs in various species possess characteristic conserved Cys residues reminiscent of Fe-S cluster binding structures. We revealed that one such MnmA in Thermus thermophilus indeed contains an oxygen-sensitive [4Fe-4S]-type cluster, which is required for $s^{2} \mathrm{U} 34$ formation.

\section{RESULTS AND DISCUSSION}

\section{Sequence analyses of MnmA homologs}

We analyzed MnmA sequences from bacteria and eukaryotes in detail and found that the MnmA homologs can be divided into two groups (Fig. 1C,D; Supplemental Fig. S1). These groups are defined by the presence of $\mathrm{C}-\mathrm{DxxC}-\mathrm{C}$ or CC-CxxC-C motifs, and we refer to them as D- and Ctype MnmAs, respectively. C-type MnmAs were found mainly in thermophilic bacterium such as Thermus thermophilus, Thermotoga maritima, and Aquifex aeolicus, in Actinobacteria such as Mycobacterium, Corynebacterium, Streptomyces, and Actinomyces, and in Cyanobacteria. In Firmicutes, both types of MnmAs were identified, including D-type MnmAs in Bacillus, Staphylococcus, and Streptococcus and C-type MnmAs in Clostridium. Although D-type MnmAs were most commonly identified in Proteobacteria (e.g., in Escherichia and Pseudomonas), a C-type MnmA variant lacking the region containing the first CC motif was found in Campylobacter and Helicobacter. We could not find C-type MnmAs in eukaryotic mitochondria and chloroplasts. Of note, both types of MnmAs can be found within the same genus (e.g., in Clostridium), suggesting that horizontal gene transfer would have occurred in the MnmA family. For example, C-type MnmAs were identified in C. acetobutylicum, C. botulinum, C. butyricum, C. difficile, C. perfringens, and C. tetani, while Dtype MnmAs were detected in C. aceticum, C. algidicarnis, C. cadaveris, C. cellulovorans, C. putrefaciens, and C. thermopalmarium.

\section{Comparison of sequences and structures of $\mathrm{MnmA}$ and TtuA}

Thermus thermophilus MnmA (TtMnmA) possess three conserved Cys residues in $\mathrm{CxxC}-\mathrm{C}$ motifs (Cys105, Cys108, and Cys200) rather than in DxxC-C motifs (Asp99, Cys102, and Cys199) in Escherichia coli MnmA $(E c M n m A)$, which are required for $s^{2}$ U34 synthesis (Fig. 1B,C; Numata et al. 2006). MnmA consists of the catalytic amino-terminal domain and the central and carboxy- terminal domains involved in tRNA recognition (Fig. 1C, bottom panel). In the EcMnmA amino-terminal domain (Fig. 1B, top panel; Numata et al. 2006), the PP-loop, the Asp99 and Cys102 residues in the rigid $\alpha$-helix, and Cys199 in a flexible loop (shown in blue), are close together in the catalytic center. A sulfate molecule is bound to the PP-loop of EcMnmA that mimics the pyrophosphate liberated following the adenylation step. Numata et al. (2006) speculated that Asp99 acts as an acid/base to protonate/ deprotonate the adenylated U34, while the ATP bound to the PP-loop supports the formation of an adenylated intermediate. In one of the two proposed mechanisms, the $S_{\gamma}$ of the persulfide formed on Cys199 is attacked by Cys102, and a liberated bisulfide nucleophilically substitutes the adenylate group to form $s^{2} U 34$. In anothertype of $s^{2} U$ thiouridylase TtuA/Ncs6, there are conserved CxxC-C motifs (consisting of Cys130, Cys133, and Cys222 in TtTtuA) in the catalytic domain, and this domain is sandwiched between the amino- and carboxy-terminal Zn-finger domains, which may contribute to tRNA binding (Fig. 1C, bottom panel; Nakagawa et al. 2013; Chen et al. 2017). Cys 130 and Cys 133 are located in the rigid $\alpha$-helix, while Cys222 is present in a flexible loop region (Fig. 1B, bottom panel). These three Cys residues bind a [4Fe-4S] cluster with a free coordination site on one of the iron atoms (Arragain et al. 2017; Chen et al. 2017). We have proposed that this iron atom binds either sulfur-carrier proteins (e.g., TtuB-COSH) or bisulfide, and that the sulfur atom is ultimately incorporated into $s^{2} U 54$.

We built a homology model of TtMnmA (Fig. 1B, middle panel) by SWISS-MODEL (Waterhouse et al. 2018) using EcMnmA as a template. The sequence identity (43\%) between TtMnmA and EcMnmA was sufficient for model building and OMEAN Z-score of TtMnmA model was -1.20 , indicating the high reliability of this model (Benkert et al. 2011). Strikingly, the model of the TtMnmA catalytic center has an architecture composed of a PP-loop and three Cys residues located in similar positions to those in TtTtuA (Fig. 1B, bottom panel). Considering this significant similarity, we speculated that MnmA homologs with $\mathrm{CxxC}-\mathrm{C}$ motifs can bind Fe-S clusters, which are required for the sulfurtransferase activity.

\section{T. thermophilus tRNAs contain $\mathrm{mnm}^{5} \mathrm{~s}^{2} \mathrm{U}$}

The residues at position 34 of the Glu, Gln, and Lys tRNAs are universally modified to 5-methyl-2-thiouridine derivatives in bacteria and eukaryotes. 5-methylaminomethyl-2thiouridine $\left(\mathrm{mnm}^{5} \mathrm{~s}^{2} \mathrm{U}\right)$ and 5-carboxymethylaminomethyl-2-thiouridine $\left(\mathrm{cmnm}^{5} \mathrm{~s}^{2} \mathrm{U}\right)$ are found in bacterial tRNAs (Carbon et al. 1968; Oashi et al. 1970; Hagervall et al. 1987). We analyzed the total nucleoside of $T$. thermophilus tRNA mixtures by LC/MS (Supplemental Fig. S2). Both $m n m^{5} s^{2} U(m / z=304.1)$ and $m^{2} m^{5} U(m / z=288.1)$ were detected, and their MS/MS spectra further confirmed 
their identities (Supplemental Fig. S2A), based on a comparison with E. coli RNA (Supplemental Fig. S2B). Neither $\mathrm{cmnm}^{5} \mathrm{~s}^{2} U(\mathrm{~m} / \mathrm{z}=348.1)$ nor $\mathrm{cmnm}^{5} U(\mathrm{~m} / \mathrm{z}=$ 332.1) was detected in T. thermophilus tRNA.

\section{T. thermophilus MnmA can bind an oxygen-labile [4Fe-4S]-type cluster}

We investigated T. thermophilus MnmA from a spectroscopic standpoint. TtMnmA expressed in E. coli and purified under aerobic conditions did not yield an ultravioletvisible (UV-VIS) spectrum which indicates the presence of a $\mathrm{Fe}-\mathrm{S}$ cluster. When aerobically purified apo-TtMnmA was incubated with iron and sulfide under anaerobic conditions, a brown solution of the TtMnmA protein was obtained and it exhibited a UV-VIS spectrum with a shoulder at $\sim 410$ $\mathrm{nm}$ (Fig. 2A), which is characteristic of [4Fe-4S] clusters in proteins (Arragain et al. 2017; Chen et al. 2017). The Fe content of the reconstituted TtMnmA sample was $3.72 \pm 0.03$ mol per mol of protein $(n=3)$, further supporting the presence of a [4Fe-4S]-type Fe-S cluster.

To clarify the properties of the Fe-S center in detail, we analyzed reconstituted $\mathrm{MnmA}$ by electron paramagnetic resonance (EPR) spectroscopy. Reconstituted MnmA displayed some small signals around $g \sim 2$ at $12 \mathrm{~K}$ that are consistent with a $[3 \mathrm{Fe}-4 \mathrm{~S}]^{1+}$ cluster and radicals (Fig. 2B [a]). After reduction with excess dithionite, the absorbance at $\sim 410 \mathrm{~nm}$ decreased (Fig. 2A). The reduced MnmA showed slightly rhombic EPR signals with principle $g=$ $[2.04,1.93,1.90]\left(g_{\mathrm{av}}=1.96\right)$ (Fig. 2B[a]), and these signals were not observed at $40 \mathrm{~K}$ (Supplemental Fig. S3A), suggesting that MnmA has a [4Fe-4S]-type cluster like those of TtuA (Arragain et al. 2017; Chen et al. 2017), aconitase (Emptage et al. 1983), and radical S-adenosylmethionine enzymes (Broderick et al. 2014; Hanson and Berliner 2017). The signal was fitted well with the simulated [4Fe$4 \mathrm{~S}]^{1+}$ cluster (dotted line) and the concentration of the $[4 \mathrm{Fe}-4 \mathrm{~S}]^{1+}$ cluster was estimated to $\sim 30 \mu \mathrm{M}$ based on comparison with double integration of the signal for the $\mathrm{Cu}^{2+}$ standard. The weak signal intensity might reflect the difficulty to reduce the cluster with dithionite (Wong et al. 1977; Vollmer et al. 1983; Henshaw et al. 2000). When ferricyanide was added to the reconstituted $\mathrm{MnmA}$, the absorbance at around $410 \mathrm{~nm}$ was also decreased (Fig. 2A). EPR signal centered at $g \sim 2$ appeared (Fig. 2B[a]), and this signal was present at $40 \mathrm{~K}$ (Supplemental Fig. S3B). The signal was not consistent with typical $[3 \mathrm{Fe}-4 \mathrm{~S}]^{1+}$ (Kennedy et al. 1992; Telser et al. 2000; Agarwalla et al. 2004) or high-potential $[4 \mathrm{Fe}-4 \mathrm{~S}]^{3+}$ signals (Dilg et al. 1999); however, this type of signal is commonly observed when ferricyanide is added to $\mathrm{Fe}-\mathrm{S}$ proteins and is generally thought to result from coupling of free radicals with iron centers (Sweeney et al. 1975; Morgan et al. 1985; Agarwalla et al. 2004). In addition, the difference spectrum between 12 and $40 \mathrm{~K}$ for ferricyanide-treated MnmA showed an obvious albeit small signal typical of $[3 \mathrm{Fe}-4 \mathrm{~S}]^{1+}(g=[2.02,2.00]$; Supplemental Fig. S3B). To confirm the degradation of the MnmA Fe-S cluster by ferricyanide oxidation, we collected EPR spectra at lower fields (Fig. 2B[b]). Oxidized MnmA showed a strong EPR signal at $g \sim 4.3$, whose intensity increased by approximately 10-fold compared with that of reconstituted MnmA, suggesting that free ferric iron was generated after the addition of ferricyanide. These EPR experiments provide evidence that reconstituted MnmA has a [4Fe4S]-type cluster, and that it is easily decomposed through oxidation.

\section{The T. thermophilus MnmA Fe-S cluster is required for $s^{2}$ U34 synthesis in tRNAs}

Because TtMnmA can bind an oxygen-labile $[4 \mathrm{Fe}-4 \mathrm{~S}]^{2+}$ cluster, we investigated the requirement of the $\mathrm{Fe}-\mathrm{S}$ cluster for its sulfurtransferase activity in vitro. We reconstituted the 2-thiolation reaction of the transcribed tRNA ${ }^{\text {Lys }}$ under anaerobic conditions in the presence of holoMnmA, $0.1 \mathrm{mM}$ sodium sulfide, and $2.5 \mathrm{mM}$ ATP (Fig. 2C). Nucleoside analysis of the tRNA reaction products by HPLC indicated the formation of $\mathrm{s}^{2} \mathrm{U}(\sim 18.5 \mathrm{~min})(\mathrm{a})$, which was confirmed by observing it had the same retention time (b) and UV spectrum as authentic $s^{2} U$ (Fig. 2D). Under these conditions, MnmA catalyzed the $s^{2} U$ formation reaction with multiple turnovers. The initial velocity of this reaction was $0.537 \pm 0.052 \mathrm{pmol} \mathrm{s}^{2} \mathrm{U} / \mathrm{pmol} \mathrm{MnmA} /$ $\min (n=3)$. A tRNA ${ }^{\text {Lys }}$ U34A mutant was not a MnmA substrate (Fig. $2 \mathrm{C}[\mathrm{c}]$ ), supporting the formation of $\mathrm{s}^{2} \mathrm{U}$ at position 34 in wild-type tRNA ${ }^{\text {Lys }}$. When we used apo-MnmA (d), no $s^{2} U$ formation was detected. The reaction also required ATP (e) and sodium sulfide ( $f$ ) in the reaction mixture. We also observed $s^{2} U 34$ formation in transcribed tRNA $^{\text {Gln }}$ (Fig. 2E). The initial velocity was $0.421 \pm 0.013$ pmol s${ }^{2} \mathrm{U} / \mathrm{pmol} \mathrm{MnmA} / \mathrm{min}(n=3)$, which is comparable $(\sim 80 \%)$ to that for tRNA ${ }^{\text {Lys }}$.

In order to examine the incorporation of exogenous sulfur into $s^{2} U$, a coupled reaction with $\left[{ }^{35} \mathrm{~S}\right]$-cysteine and cysteine desulfurase with holo-MnmA was performed, and the incorporation of $\left[{ }^{35} \mathrm{~S}\right]$-sulfur in tRNA was readily detected (Fig. 2F). The $\left[{ }^{35} \mathrm{~S}\right]$-persulfide formed on the catalytic Cys residue $\left(\mathrm{R}-\mathrm{S}\left[{ }^{35} \mathrm{~S}\right] \mathrm{H}\right)$ in the cysteine desulfurases was released as $\left.{ }^{35} \mathrm{~S}\right]$-sulfide by dithiothreitol (DTT) in the reaction mixture (Behshad et al. 2004), and the released $\left[{ }^{35} \mathrm{~S}\right]$-sulfide could be further utilized and incorporated into tRNA by holo-MnmA. Taken together, these data demonstrate that the MnmA Fe-S cluster is required for $s^{2} U 34$ synthesis in tRNAs. These results also suggest that exogenous sulfur (i.e., bisulfide), rather than the sulfur atom of the Fe-S cluster, is incorporated into $\mathrm{s}^{2} \mathrm{U}$. In addition, TtMnmA does not require $\mathrm{C} 5$-modification of $\mathrm{U} 34$ for $s^{2} U$ synthesis, because the transcript tRNAs were good substrates for TtMnmA. 
A

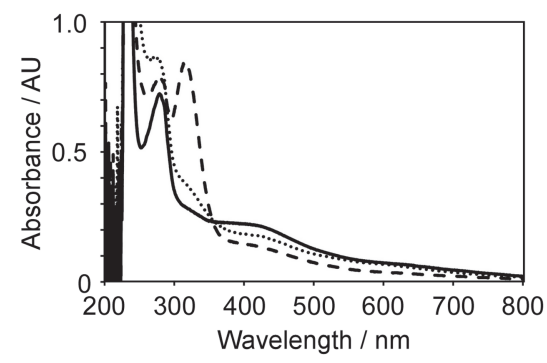

C
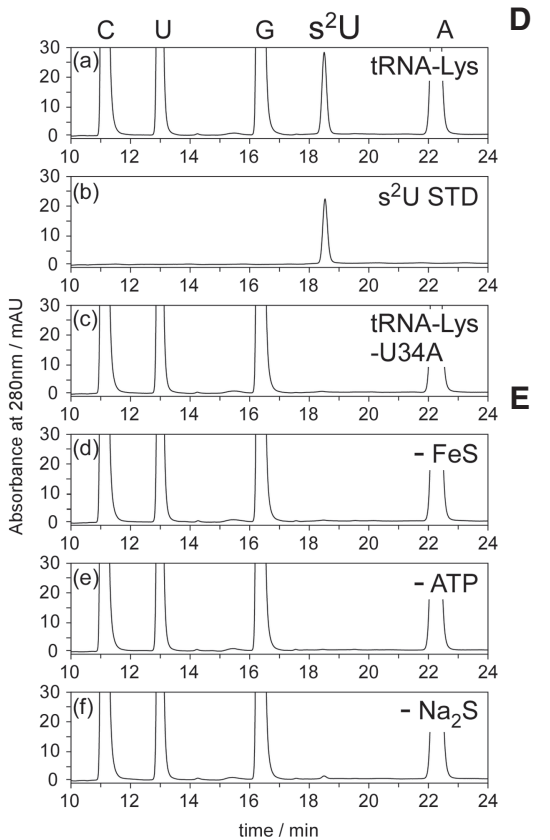

B (a)

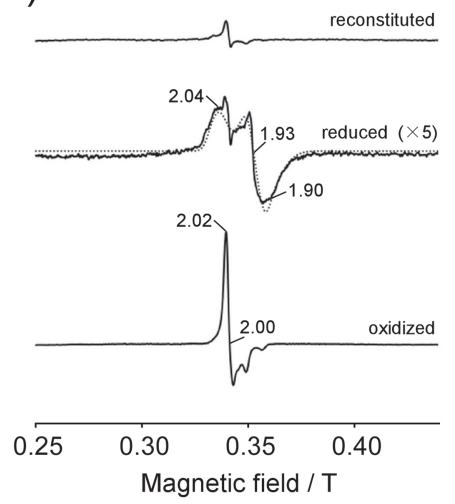

(b)

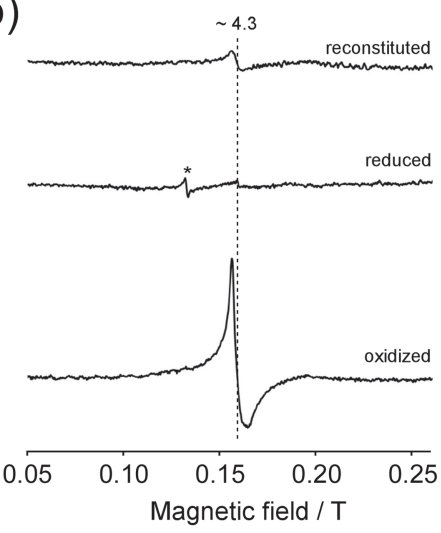

$\mathbf{F}$
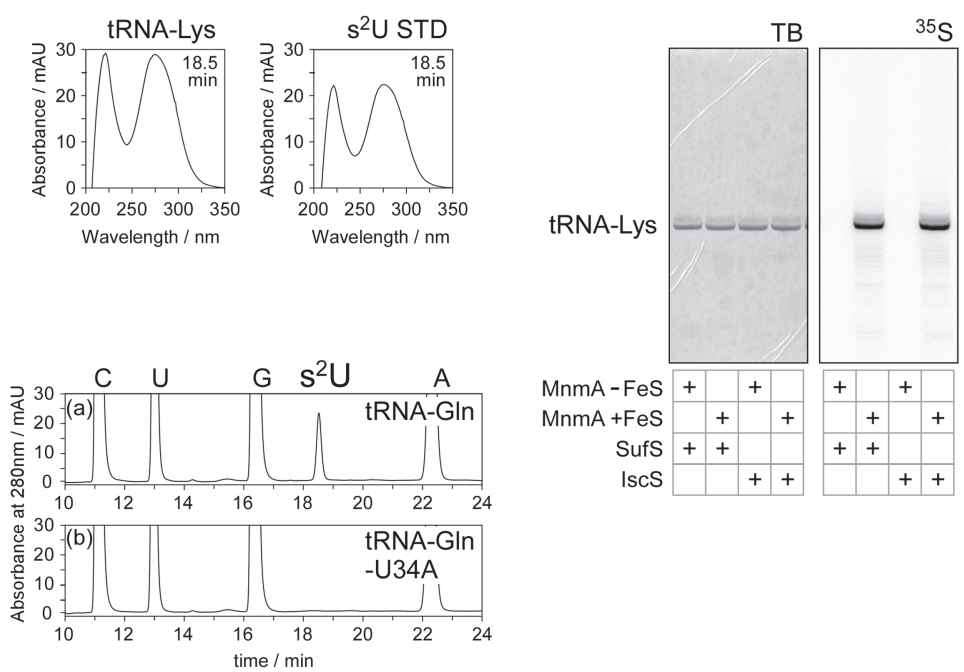

FIGURE 2. Characterization of T. thermophilus MnmA. (A) UV-VIS spectra of reconstituted (solid line), reduced (dashed line), and oxidized MnmA (dotted line). The samples of reduced and oxidized MnmA were prepared by adding 10 eq of sodium dithionite and ferricyanide solution, respectively, and diluted to $0.7 \mathrm{mg} / \mathrm{mL}$ in A buffer. The peak at around $310 \mathrm{~nm}$ in the reduced sample was derived from excess dithionite. (B) EPR spectra of reconstituted, reduced, and oxidized $\mathrm{MnmA}(\sim 0.7 \mathrm{mM})$ at $12 \mathrm{~K}$ in high (a) and low (b) magnetic fields. The principle $g$-values are shown in the figure. All spectra acquired under a high magnetic field were subjected to subtraction of the spectrum acquired using buffer alone. A simulated spectrum of the $[4 \mathrm{Fe}-4 \mathrm{~S}]^{1+}$ cluster is also presented in (a), represented by a dotted line. The asterisk in (b) shows an unknown signal with $g \sim 5.7$. Detailed conditions: microwave frequency $=\sim 9.59 \mathrm{GHz}$, microwave power $=1 \mathrm{~mW}, 100 \mathrm{kHz}$ modulation amplitude $=10 \mathrm{G}$, time constants $=41$ $\mathrm{ms}$, number of scans $=4$, and temperature $=12 \mathrm{~K}$. (C) Nucleoside analysis of transcribed tRNA ${ }^{\text {Lys }}$ from in vitro reactions with holo-TtMnmA. The modified nucleosides of the reacted RNA were analyzed by HPLC. (a) tRNA ( 450 pmol) was reacted at $60^{\circ} \mathrm{C}$ for 20 min with 19 pmol of MnmA in the presence of $0.1 \mathrm{mM}$ sodium sulfide, $2.5 \mathrm{mM}$ ATP, and $0.1 \mathrm{mM}$ DTT. (b) $\mathrm{s}^{2} U$ standard. (c) tRNA ${ }^{\text {Lys }}$ with a U34A mutation was used. (d) ApoMnmA was used. In (e) and (f), ATP and sodium sulfide were omitted, respectively. (D) UV spectra of $s^{2} U$ detected at 18.5 min in tRNA ${ }^{\text {Lys }}(C$ [a]) and authentic $s^{2} U(C[b])$. (E) Nucleoside analysis of transcribed tRNA ${ }^{\text {Gln }}$ from in vitro reactions. Wild-type (a) and U34A mutant (b) molecules were analyzed. The assay conditions were the same as in $C$. (F) $\left[{ }^{35} \mathrm{~S}\right]$-Cys-derived ${ }^{35} \mathrm{~S}$-sulfur incorporation into $\mathrm{s}^{2} \mathrm{U}$. tRNA ${ }^{\text {Lys }}$ (450 pmol) was reacted at $60^{\circ} \mathrm{C}$ for $30 \mathrm{~min}$ with $19 \mathrm{pmol}$ of apo- or holo-MnmA and $75 \mathrm{pmol}$ of IscS or SufS in the presence of $20 \mu \mathrm{M}\left[{ }^{35} \mathrm{~S}\right]-\mathrm{Cys}, 20 \mu \mathrm{M}$ pyridoxal phosphate, $2.5 \mathrm{mM}$ ATP, and $0.1 \mathrm{mM}$ DTT. The reacted RNA was separated by $10 \%$ denaturing PAGE and then stained with toluidine blue (TB; left), after which the ${ }^{35} \mathrm{~S}$ radioactivity was visualized (right).

\section{Mutational analysis of conserved residues in TtMnmA}

A comparison of the structure of the TtMnmA model with that of TtTtuA (Fig. 1B) suggested that the TtMnmA [4Fe$4 \mathrm{~S}$ ] cluster is coordinated by three conserved Cys residues:
Cys105, Cys108, and Cys200. To evaluate the importance of these Cys residues, we reconstituted Fe-S clusters in a series of Ala-substituted TtMnmA mutants (Supplemental Fig. S5A) and measured their Fe content and enzymatic activities (Table 1). As expected, the Fe content was 
TABLE 1. In vitro mutational analysis of TtMnmA

\begin{tabular}{|c|c|c|c|}
\hline & \multirow{2}{*}{$\begin{array}{l}\text { Amount of Fe } \\
(\text { pmol Fe/ } \\
\text { pmol MnmA })^{a}\end{array}$} & \multicolumn{2}{|c|}{ Enzyme activity } \\
\hline & & $\begin{array}{c}\left(\mathrm{pmol} \mathrm{s} \mathrm{s}^{2} \mathrm{U} / \mathrm{pmol}\right. \\
\mathrm{MnmA} / \mathrm{min})^{\mathrm{a}}\end{array}$ & $\%^{\mathrm{b}}$ \\
\hline Wild-type & $3.72 \pm 0.03$ & $0.567 \pm 0.078$ & 100.0 \\
\hline C105A & $3.32 \pm 0.21$ & $0.007 \pm 0.000$ & 1.3 \\
\hline C108A & $3.02 \pm 0.12$ & Not detected & 0 \\
\hline C200A & $3.49 \pm 0.15$ & $0.195 \pm 0.013$ & 34.4 \\
\hline $\begin{array}{l}\text { C105A, C108A, } \\
\text { C200A }\end{array}$ & $1.96 \pm 0.08$ & Not detected & 0 \\
\hline D13A & $3.73 \pm 0.05$ & Not detected & 0 \\
\hline C55A & $3.65 \pm 0.25$ & $0.614 \pm 0.009$ & 108.3 \\
\hline C56A & $3.83 \pm 0.12$ & $0.957 \pm 0.048$ & 168.7 \\
\hline C55A, C56A & $3.57 \pm 0.22$ & $0.609 \pm 0.021$ & 107.4 \\
\hline
\end{tabular}

decreased in each of these mutants (Table 1), and the shoulder peaking at $\sim 410 \mathrm{~nm}$ in the UV-VIS spectrum was also diminished (Supplemental Fig. S5B), especially in the triple mutant. The relatively large amount of $\mathrm{Fe}$ in the triple mutant relative to the wild-type protein may be due to nonspecific binding of $\mathrm{Fe}$ being enhanced by the loss of a binding site for the genuine $\mathrm{Fe}-\mathrm{S}$ cluster. To evaluate the stability of the Fe-S cluster of reconstituted samples, the $A_{410} / A_{280}$ ratios were measured after $10 \mathrm{~d}$ of storage at $20^{\circ} \mathrm{C}$ in an anaerobic chamber. The $A_{410} /$ $\mathrm{A}_{280}$ ratios for $\mathrm{C} 105 \mathrm{~A}, \mathrm{C108 \textrm {A }}$, and C200A mutants were reduced to $70 \pm 4 \%, 62 \pm 6 \%$, and $70 \pm 1 \%$ of those values immediately after reconstitution, respectively, although the $A_{410} / A_{280}$ ratios of wild-type TtMnmA retain $82 \pm 4 \%$ of the initial value. These results suggest that the stability of the $\mathrm{Fe}-\mathrm{S}$ cluster is reduced in these mutants. In addition, the UV-VIS spectrum of C105A and C108A mutants also exhibited broad shoulder at $\sim 600 \mathrm{~nm}$ in addition to $\sim 410 \mathrm{~nm}$ region observed in wild-type TtMnmA (Supplemental Fig. S5B), suggesting there exists subtle differences in $\mathrm{Fe}-\mathrm{S}$ clusters between the mutants and the wild-type. Consistent with these observations, the 2-thiolation activities of the single mutants were also decreased, and this activity was completely abolished in the triple mutant (Table 1). These data suggest that the Fe-S cluster is chelated by the Cys105, Cys108, and Cys200 residues, and that it is essential for 2-thiolation activity.

Asp13 is a strictly conserved residue in the PP-loops of C- and D-type MnmAs. TtMnmA carrying a D13A mutation bound nearly the same amount of Fe (Table 1) and exhibited nearly the same UV-VIS spectra as the wild-type protein (Supplemental Fig. S5B), suggesting that this mutation did not affect the binding of the Fe-S cluster. Otherwise, the 2-thiolation activity of the D13A mutant was complete- ly abolished, supporting the importance of Asp13, which may be involved in the formation of adenylate.

These mutational analyses support a putative two-step reaction mechanism (Fig. 1A): (i) TtMnmA first activates the $\mathrm{C} 2$ position of U34 via adenylation, as seen in related PP-loop ATP pyrophosphatases, such as the previously characterized D-type EcMnmA (Numata et al. 2006) and tRNA-lysidine synthetase TilS (lkeuchi et al. 2005); (ii) subsequently, a sulfide group bound to an iron atom in a free coordination site of the [4Fe-4S] cluster is incorporated into $s^{2} \cup 34$, similar to the proposed mechanism for the TtuA/Ncs6 family sulfurtransferases (Arragain et al. 2017; Chen et al. 2017), in sharp contrast to the mechanism of D-type EcMnmA in this second step (see above) (Numata et al. 2006). This proposal needs to be validated by additional experiments.

We also investigated two additional conserved Cys residues in TtMnmA: Cys55 and Cys56. The two Cys residues are highly conserved in C-type MnmAs (Fig. 1C), while Dtype Fe-S-independent MnmAs contain only one Cys residue. Moreover, there is a variant of C-type MnmA that does not contain these Cys residues. The Fe content and UV-VIS spectra of the C55A, C56A, and C55A:C56A mutants were nearly the same as those of the wild-type protein (Table 1; Supplemental Fig. S5B). Although the 2-thiolation activities of the C55A and C55A:C56A mutants were nearly the same as those of the wild-type protein, the activity of the C56A mutant was increased by approximately 1.7 -fold. These results indicate that these Cys residues are dispensable for the 2-thiolation reaction; however, the basis of the enhanced reaction in the C56A mutant remains unclear.

\section{Cysteine desulfurases can supply sulfur to TtMnmA}

In E. coli, the sulfur atom of cysteine is first activated by the IscS cysteine desulfurase to form a protein-persulfide IscS-SSH derivative (Flint 1996; Ikeuchi et al. 2006). The persulfide group is relayed by the TusA, TusBCD, and TusE carrier proteins and is passed onto the catalytic Cys199 residue in MnmA; finally, the sulfur atom is incorporated into $s^{2} \cup 34$. In B. subtilis (Black and Dos Santos 2015), the BsYrvO cysteine desulfurase and BsMnmA are necessary and sufficient for $\mathrm{s}^{2} \mathrm{U} 34$ synthesis. Although cysteine desulfurases, TusA, and MnmA are conserved in many organisms, TusBCD and TusE are not conserved (Kotera et al. 2010), implying that variations exist in the upstream sulfur-transfer pathways for $\mathrm{s}^{2} U$ synthesis.

Two cysteine desulfurases, IscS and SufS, are encoded by the T. thermophilus genome (Shigi et al. 2006a), and no homologs of the Tus proteins have been identified, suggesting direct transfer of sulfur from the cysteine desulfurases to MnmA, although indirect sulfur transfer via unidentified sulfur-carrier protein(s) could exist. Therefore, we investigated $s^{2} \cup 34$ formation in vitro by cysteine 
desulfurases with cysteine as the sulfur donor. In this assay, the reductants included in the enzyme stock solutions were eliminated by gel filtration to prevent possible sulfide release into the reaction mixture. In the absence of reductant, $s^{2} U$ was readily formed by the cysteine desulfurases using cysteine as the sulfur donor. The initial velocities were $0.136 \pm 0.010$ and $0.329 \pm 0.013\left(\mathrm{pmol} \mathrm{s} \mathrm{s}^{2} \mathrm{U} / \mathrm{pmol}\right.$ $\mathrm{MnmA} / \mathrm{min}, \mathrm{n}=3$ ) for $1.25 \mu \mathrm{M}$ IscS and SufS with $0.1 \mathrm{mM}$ cysteine, respectively. These results suggest direct persulfide transfer from the cysteine desulfurases to MnmA during $s^{2} U$ formation.

\section{Concluding remarks}

For $s^{2} U 34$ formation in tRNAs, there are many C-type MnmA homologs (Figs. 1C,D, 3) that likely possess Fe-S clusters similar to that of TtMnmA, in addition to the previously characterized DxxC-C-type (D-type, non-FeS) MnmAs. As C-type MnmAs are found in many thermophilic bacteria (Fig. 1C,D), which are generally considered to be closely related to the common ancestor of life (Akanuma et al. 2013), C-type MnmA seems to be an ancient member of the MnmA family. Figure 3 summarizes the distribution of the $s^{2} U$ thiouridylases in the three domains of life. In the cytoplasm of eukaryotes and archaea, $s^{2} U 34$ is formed by the Ncs6 and NcsA Fe-S proteins (Liu et al. 2016). Furthermore, in $T$. thermophilus, $s^{2}$ U54 is synthesized by the TtuA Fe-S protein. Ncs6/NcsA/TtuA possess catalytic centers similar to that of C-type $\mathrm{MnmA}$, although they may bind tRNAs differently by distinct RNA binding domains (Fig. 1C). Many archaea carry two ORFs related to NcsA/TtuA (Shigi et al. 2006a; Chavarria et al. 2014), suggesting that they may be involved in the biosynthesis of $s^{2} U 34$ and $s^{2}$ U54; however, experimental validation is needed. Another important issue that remains to be addressed is whether a sulfide group bound to an iron atom in a free coordination site of the [4Fe-4S] cluster is truly incorporated into $s^{2} U$.

In some cases of the PP-loop ATP pyrophosphatases family, the Fe-S-dependent and Fe-S-independent enzymes possess the same enzymatic activities. Although most Thil homologs responsible for 4-thiouridine synthesis at position 8 of tRNAs are Fe-S cluster-independent enzymes, some archaeal (e.g., Methanogenic archaea and Thermococcales) Thil homologs have conserved $\mathrm{CxxC}$ C motifs (Liu et al. 2012), and are essential for their [3Fe4S] cluster binding and enzymatic activity (Liu et al. 2016). It is quite intriguing that loss or acquisition of FeS clusters may occur via changes in one or two Cys residues and that these changes seem to have occurred independently in several enzyme groups and even in orthologous enzymes in closely related species, suggesting a general evolutionary strategy for enzymes with several sulfur-transfer activities. The oxygen sensitivity of Fe-S proteins may be compensated for by universally conserved iron-sulfur cluster repair systems in the cells (Zheng and Dos Santos 2018), which support wide-spread utilization of these oxygen-sensitive sulfurtransferases and no definite correlation with habitat environments (e.g., anaerobic or aerobic).

Because $s^{2} U 34$ moieties in tRNA have quite important roles for translation and defects in $s^{2} U 34$ synthesis lead to severe growth retardation or death (Kambampati and Lauhon 2003; Ikeuchi et al. 2006; Bjork et al. 2007; Dewez et al. 2008; Chavarria et al. 2014; Black and Dos Santos 2015), s² U34 biosynthesis is a good target for antibiotic development. The different architectures in their catalytic centers and domain structures of $s^{2} U 34$ thiouridylases could provide a basis for the development of novel antibiotics, as specific inhibitors of the C-type MnmAs of pathogens are unlikely to inhibit the eukaryotic counterparts.

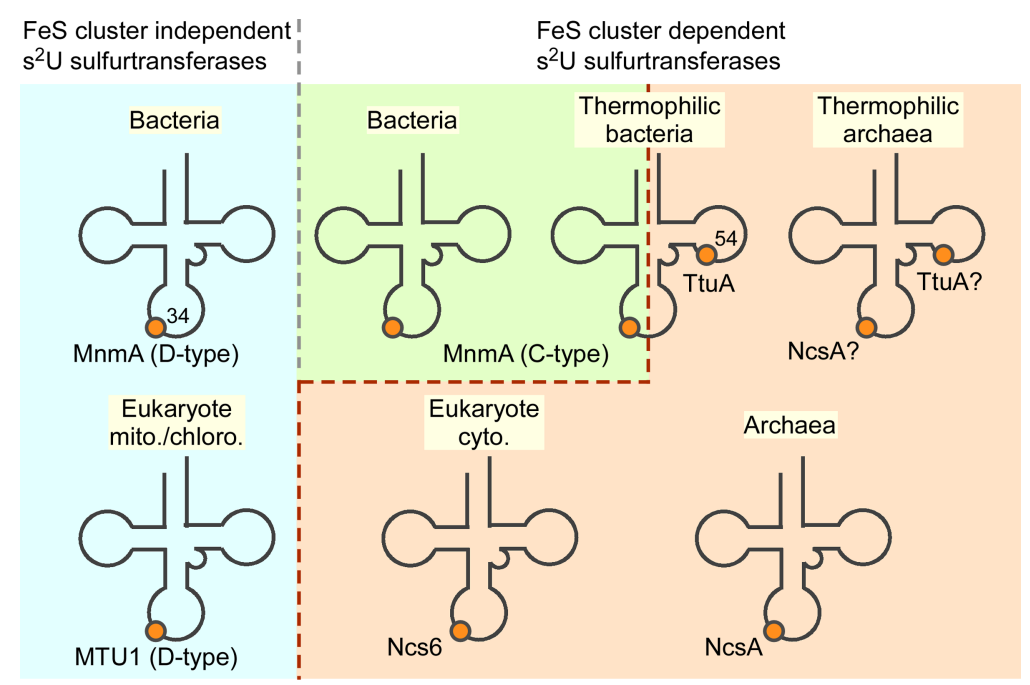

FIGURE 3. Sulfur transferases involved in $s^{2} U$ synthesis in the three domains of life. In this study, it was found that a C-type TtMnmA is a Fe-S protein. See main text for details.

\section{MATERIALS AND METHODS}

\section{Recombinant proteins}

The expression plasmid for MnmA was pET22b-TtMnmA. The gene encoding $T$. thermophilus HB27 MnmA (Ttc1727) was cloned into the Ndel and Hindlll sites of pET22b (Novagen), resulting in the generation of a carboxy-terminally $\mathrm{His}_{6}$-tagged fusion protein. The expression plasmid for the Ala variants was constructed by site-directed mutagenesis (QuikChange II Mutagenesis Kit, Stratagene). The sequences of the plasmids were confirmed by sequencing. The primers used are listed in Supplemental Table S1. The wild-type and variants of the $\mathrm{His}_{6}$-tagged TtMnmA were expressed in the Rosetta2 DE3 E. coli strain (Novagen) and purified under 
aerobic conditions. Cultures were grown to an $\mathrm{OD}_{600}$ of approximately 0.6 , induced with $0.5 \mathrm{mM}$ isopropyl 1 -thio- $\beta$-D-galactopyranoside, and then incubated for an additional $6 \mathrm{~h}$ at $37^{\circ} \mathrm{C}$. Cells were lysed for $\sim 30 \mathrm{~min}$ at $25^{\circ} \mathrm{C}$ in A buffer [50 mM Hepes- $\mathrm{KOH}$ (pH 7.6),

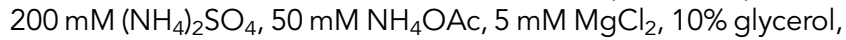
and $7 \mathrm{mM} \beta$-mercapto-ethanol ( $\beta \mathrm{ME}$ )] containing $1 \times$ bugbuster reagent (Novagen) and $0.2 \mathrm{mM}$ phenylmethylsulfonyl fluoride. The cell debris were then removed by centrifugation. The TtMnmA in the cleared lysate was bound to Ni-NTA agarose (Qiagen), washed with A buffer containing $1 \mathrm{M} \mathrm{NH}_{4} \mathrm{Cl}$ and $10 \mathrm{mM}$ imidazole, and eluted with A buffer containing $250 \mathrm{mM}$ imidazole. The purified proteins were desalted using NAP 25 gel filtration columns (GE Healthcare) with A buffer. The TtMnmA samples purified under aerobic conditions were nearly colorless (apo-TtMnmA). Protein concentrations were determined using a Bio-Rad protein assay kit with bovine serum albumin as a standard. Amino-terminal $\mathrm{His}_{6}$-tagged versions of $\mathrm{T}$. thermophilus IscS and SufS were purified as described previously (Shigi et al. 2006a).

\section{Reconstitution of the TtMnmA iron-sulfur cluster}

All manipulations were performed under strictly anaerobic conditions ( $\left.<2 \mathrm{ppm}\left[\mathrm{O}_{2}\right]\right)$ in an anaerobic glove box (COY) under an atmosphere of $95 \% \mathrm{~N}_{2}$ and $5 \% \mathrm{H}_{2}$ with a palladium catalyst. Solutions were made anoxic by bubbling for a minimum of 3 h. Apo-TtMnmA (0.03 mM) purified under aerobic conditions was incubated for $3 \mathrm{~h}$ at $25^{\circ} \mathrm{C}$ in A buffer containing $1.5 \mathrm{mM}$ DTT, $0.3 \mathrm{mM} \mathrm{FeCl}_{3}$, and $0.3 \mathrm{mM} \mathrm{Na}_{2} \mathrm{~S}$. Excess $\mathrm{FeCl}_{3}$ and $\mathrm{Na}_{2} \mathrm{~S}$ were removed using NAP 5 gel filtration columns (GE Healthcare) with A buffer, and the reconstituted TtMnmA was concentrated using $30 \mathrm{kDa}$-cutoff Amicon Ultra filters (MerckMillipore). Protein concentrations were determined using a BioRad protein assay kit, and the Fe concentrations of the protein samples were determined by a colorimetric method with $\mathrm{FeSO}_{4}$ as a standard (BioChain) (Hoppe et al. 2003). The UV-VIS spectra of the protein samples were recorded using a UV-1800 spectrophotometer (Shimadzu). All of the samples were stored at $20^{\circ} \mathrm{C}$ and kept anoxic in a glove box.

\section{EPR spectroscopy}

Anaerobically reconstituted TtMnmA was concentrated to $\sim 0.7$ $\mathrm{mM}$ and then reduced or oxidized via the addition of $10 \mathrm{eq}$ of sodium dithionite or potassium ferricyanide, respectively. Continuous-wave X-band EPR spectra were collected using a Bruker ELEXSYS E580 spectrometer operating at $\sim 9.59 \mathrm{GHz}$, equipped with an Oxford Instruments ESR 910 continuous helium flow cryostat (Analytical Research Center for Experimental Sciences, Saga University). Other experimental parameters are indicated in the figure legends. Simulation of EPR spectra was performed using the EasySpin 5.2.20 program (Stoll and Schweiger 2006) in the MATLAB environment.

\section{In vitro tRNA transcription}

tRNA $^{\text {Lys }}$ and tRNA ${ }^{\text {Gln }}$ (with a U1G mutation for efficient transcription) were prepared by in vitro transcription using T7 RNA polymerase (Milligan and Uhlenbeck 1989). Templates for the in vitro transcription were constructed by PCR using synthetic oligonucleotide DNAs carrying the tRNA genes under the control of the T7 promoter sequence. The PCR products were cloned into the EcoRI and Hindlll sites of pUC18. Plasmids for the U34A mutants were constructed by site-directed mutagenesis (QuikChange II Mutagenesis Kit, Stratagene). For the in vitro transcription reactions, primer pairs were used to PCR-amplify the template plasmid. The primers used are listed in Supplemental Table S1. The transcripts of the tRNA genes were prepared at $37^{\circ} \mathrm{C}$ for $3 \mathrm{~h}$ in a reaction mixture containing $40 \mathrm{mM}$ HEPES$\mathrm{KOH}$ (pH 7.8), $8 \mathrm{mM} \mathrm{MgCl}$, $5 \mathrm{mM}$ DTT, $1 \mathrm{mM}$ spermidine, 1 $\mathrm{mM}$ of each NTP, $5 \mathrm{mM}$ GMP, $50 \mu \mathrm{g} / \mathrm{mL}$ bovine serum albumin, $2 \mu \mathrm{g} / \mathrm{mL}$ template DNA, and T7 RNA polymerase (Milligan and Uhlenbeck 1989). RNA was extracted from the reaction mixture by the acid-guanidinium thiocyanate-phenol-chloroform reagent (Isogen, Wako) and precipitated with 2-propanol. Next, the reaction for CCA addition was performed as described previously (Tomari et al. 2000). The transcripts of the tRNA genes were treated with E. coli CCA-adding enzyme at $37^{\circ} \mathrm{C}$ for $30 \mathrm{~min}$ in a reaction mixture containing $50 \mathrm{mM}$ HEPES-KOH ( $\mathrm{pH} 7.6), 100 \mathrm{mM}$ $\mathrm{KCl}, 10 \mathrm{mM} \mathrm{MgCl} 2,2 \mathrm{mM}$ DTT, $1 \mathrm{mM} \mathrm{ATP}$, and $0.2 \mathrm{mM} \mathrm{CTP.}$ The RNA was recovered using Isogen, precipitated by 2-propanol, and further purified using denaturing 10\% PAGE.

\section{In vitro $s^{2} U$ formation with holo-TtMnmA}

$s^{2} U$ formation on substrate tRNA was assayed under strictly anaerobic conditions in a glove box. The standard assay was performed at $60^{\circ} \mathrm{C}$ for $5-30 \mathrm{~min}$ in $60 \mu \mathrm{L}$ of $\mathrm{H}$ buffer $(50 \mathrm{mM}$ Hepes- $\mathrm{KOH}$ [pH 7.6], $100 \mathrm{mM} \mathrm{KCl}, 10 \mathrm{mM} \mathrm{MgCl}_{2}$, and $0.1 \mathrm{mM} \mathrm{DTT}$ ) containing 2.5 $\mathrm{mM}$ ATP, $0.1 \mathrm{mM} \mathrm{Na} \mathrm{S}_{2}, 450$ pmol of tRNA, and 9.4-75 pmol of holo-MnmA. For the cysteine desulfurase coupled reactions, 0.1 $\mathrm{mM}$ Cys, $20 \mu \mathrm{M}$ pyridoxal phosphate, and 75 pmol of IscS or SufS were included in the reaction mixture. For the experiment under nonreducing conditions, $\beta M E$ was removed from the enzyme stock solutions using NAP 5 gel filtration columns (GE Healthcare), and $\mathrm{H}$ buffer without DTT was used. To determine the initial velocities, the reactions were analyzed in triplicate with tRNA ${ }^{\text {Lys }}$, unless otherwise stated. After the reactions, the tRNA was recovered by Isogen (Wako) and precipitated with ethanol. Hydrolysates of thiolated tRNA were prepared and analyzed using a HPLC system with an ODS column and a photo diode array detector (GL-Sciences) as described previously (Shigi et al. 2008). The amount of $s^{2} U$ formed was quantified using authentic $s^{2} U$ (Jena Bioscience) as standard. Details of the determination of initial velocities are presented in Supplemental Figure S4.

The reaction using $\left[{ }^{35} \mathrm{~S}\right]$-Cys as the $\mathrm{S}$ donor was performed using $20 \mu \mathrm{M}\left[{ }^{35} \mathrm{~S}\right]$-Cys (6 $\mu \mathrm{Ci}$, PerkinElmer), 75 pmol of cysteine desulfurases, and $19 \mathrm{pmol}$ of holo-MnmA in the presence of 20 $\mu \mathrm{M}$ pyridoxal phosphate, $2.5 \mathrm{mM}$ ATP, and $0.1 \mathrm{mM}$ DTT. After the completion of the reactions, the recovered RNA was separated by PAGE in $10 \%$ gels containing $7 \mathrm{M}$ urea, and the gels were stained with $0.025 \%$ toluidine blue. Finally, the gels were dried, exposed on an imaging plate, and analyzed using the BAS 2500 system (Fuji Photo Systems).

\section{Analysis of tRNA modifications by LC/MS}

For the HILIC/ESI-MS analyses, a ZIC-cHILIC column ( $3 \mu \mathrm{m}$ particle size, $2.1 \times 150 \mathrm{~mm}$, Merck-Millipore) was used on a $\mathrm{Q}$ Exactive hybrid Quadrupole-Orbitrap mass spectrometer 
(Thermo Fisher Scientific) equipped with an ESI source and an Ultimate 3000 liquid chromatography system (Dionex) (Sakaguchi et al. 2015). The mobile phase consisted of $5 \mathrm{mM}$ ammonium acetate ( $\mathrm{pH} \mathrm{5.3)} \mathrm{(solvent} A$ ) and acetonitrile (solvent $B$ ). Total nucleosides were prepared by digesting 2-4 $\mu \mathrm{g}$ of a tRNA mixture from $T$. thermophilus HB27 or total RNA from E. coli BW25113 with $0.05 \mathrm{U}$ nuclease P1 (Wako) and $0.04 \mathrm{U}$ dialyzed bacterial alkaline phosphatase (C75, Wako) in $20 \mathrm{mM}$ trimethylamine-acetate buffer $\left(\mathrm{pH} \mathrm{5.3)}\right.$ at $37^{\circ} \mathrm{C}$ for $1 \mathrm{~h}$. Nucleosides dissolved in $90 \%$ acetonitrile were injected and chromatographed at a flow rate of $100 \mu \mathrm{L} / \mathrm{min}$ over a multistep linear gradient: $90 \%-80 \%$ B from 0 to $10 \mathrm{~min}, 80 \%-30 \%$ B from 10 to $30 \mathrm{~min}$, $30 \% \mathrm{~B}$ for $10 \mathrm{~min}$, and then $90 \% \mathrm{~B}$. The proton adducts of the nucleosides were scanned in a positive polarity mode $(\mathrm{m} / \mathrm{z} 110$ 900).

\section{SUPPLEMENTAL MATERIAL}

Supplemental material is available for this article.

\section{ACKNOWLEDGMENTS}

We would like to thank Dr. Toshifumi Ueda (AIST) for constructing the plasmids encoding the MnmA mutants. We would also like to thank Dr. Koh Takeuchi (AIST) for comments on this study. This study was supported in part by Grants-in-Aid for Scientific Research (16K07311 to N.S., $17 \mathrm{H06955}$ and $18 \mathrm{H} 02412$ to M.H.) from the Ministry of Education, Culture, Sports, Science, and Technology of Japan, the Precise Measurement Technology Promotion Foundation (to N.S.), the Takeda Science Foundation (to N.S.), and Sumitomo Electric Group CSR Foundation (to N.S.).

Received September 6, 2019; accepted December 1, 2019.

\section{REFERENCES}

Agarwalla S, Stroud RM, Gaffney BJ. 2004. Redox reactions of the iron-sulfur cluster in a ribosomal RNA methyltransferase, RumA: optical and EPR studies. J Biol Chem 279: 34123-34129. doi:10 .1074/jbc.M405702200

Agris PF, Soll D, Seno T. 1973. Biological function of 2-thiouridine in Escherichia coli glutamic acid transfer ribonucleic acid. Biochemistry 12: 4331-4337. doi:10.1021/bi00746a005

Agris PF, Sierzputowska-Gracz H, Smith W, Malkiewicz A, Sochacka E, Nawrot B. 1992. Thiolation of uridine carbon-2 restricts the motional dynamics of the transfer RNA wobble position nucleoside. J Am Chem Soc 114: 2652-2656. doi:10.1021/ja00033a044

Akanuma S, Nakajima Y, Yokobori S, Kimura M, Nemoto N, Mase T, Miyazono K, Tanokura M, Yamagishi A. 2013. Experimental evidence for the thermophilicity of ancestral life. Proc Natl Acad Sci USA 110: 11067-11072. doi:10.1073/pnas. 1308215110

Armengod ME, Meseguer S, Villarroya M, Prado S, Moukadiri I, RuizPartida R, Garzon MJ, Navarro-Gonzalez C, Martinez-Zamora A. 2014. Modification of the wobble uridine in bacterial and mitochondrial tRNAs reading NNA/NNG triplets of 2-codon boxes. RNA Biol 11: 1495-1507. doi:10.4161/15476286.2014.992269

Arragain S, Bimai O, Legrand P, Caillat S, Ravanat J-L, Touati N, Binet L, Atta M, Fontecave M, Golinelli-Pimpaneau B. 2017. Nonredox thiolation in tRNA occurring via sulfur activation by a [4Fe-4S] cluster. Proc Natl Acad Sci USA 114: 7355-7360. doi:10.1073/pnas.1700902114
Ashraf SS, Sochacka E, Cain R, Guenther R, Malkiewicz A, Agris PF. 1999. Single atom modification $(\mathrm{O} \rightarrow \mathrm{S})$ of tRNA confers ribosome binding. RNA 5: 188-194. doi:10.1017/S1355838299981529

Behshad E, Parkin SE, Bollinger JM Jr. 2004. Mechanism of cysteine desulfurase Slr0387 from Synechocystis sp. PCC 6803: kinetic analysis of cleavage of the persulfide intermediate by chemical reductants. Biochemistry 43: 12220-12226. doi:10.1021/bi049143e

Benkert P, Biasini M, Schwede T. 2011. Toward the estimation of the absolute quality of individual protein structure models. Bioinformatics 27: 343-350. doi:10.1093/bioinformatics/btq662

Bjork GR, Huang B, Persson OP, Bystrom AS. 2007. A conserved modified wobble nucleoside $\left(\mathrm{mcm}^{5} \mathrm{~s}^{2} \mathrm{U}\right)$ in lysyl-tRNA is required for viability in yeast. RNA 13: 1245-1255. doi:10.1261/rna.558707

Black KA, Dos Santos PC. 2015. Abbreviated pathway for biosynthesis of 2-thiouridine in Bacillus subtilis. J Bacteriol 197: 1952-1962. doi:10.1128/JB.02625-14

Boccaletto P, Machnicka MA, Purta E, Piạtkowski P, Bagiński B, Wirecki TK, de Crécy-Lagard V, Ross R, Limbach PA, Kotter A, et al. 2018. MODOMICS: a database of RNA modification pathways. 2017 update. Nucleic Acids Res 46: D303-D307. doi:10 $.1093 /$ nar/gkx1030

Bork P, Koonin EV. 1994. A P-loop-like motif in a widespread ATP pyrophosphatase domain: implications for the evolution of sequence motifs and enzyme activity. Proteins 20: 347-355. doi:10 .1002/prot.340200407

Broderick JB, Duffus BR, Duschene KS, Shepard EM. 2014. Radical Sadenosylmethionine enzymes. Chem Rev 114: 4229-4317. doi:10 $.1021 / \mathrm{cr} 4004709$

Carbon J, David H, Studier MH. 1968. Thiobases in Escherchia coli transfer RNA: 2-thiocytosine and 5-methylaminomethyl-2-thiouracil. Science 161: 1146-1147. doi:10.1126/science.161.3846.1146

Chavarria NE, Hwang S, Cao S, Fu X, Holman M, Elbanna D, Rodriguez S, Arrington D, Englert M, Uthandi S, et al. 2014. Archaeal Tuc1/Ncs6 homolog required for wobble uridine tRNA thiolation is associated with ubiquitin-proteasome, translation, and RNA processing system homologs. PLoS One 9: e99104. doi:10.1371/journal.pone.0099104

Chen M, Asai SI, Narai S, Nambu S, Omura N, Sakaguchi Y, Suzuki T, Ikeda-Saito M, Watanabe K, Yao M, et al. 2017. Biochemical and structural characterization of oxygen-sensitive 2-thiouridine synthesis catalyzed by an iron-sulfur protein TtuA. Proc Natl Acad Sci USA 114: 4954-4959. doi:10.1073/pnas.1615585114

Dewez M, Bauer F, Dieu M, Raes M, Vandenhaute J, Hermand D. 2008. The conserved Wobble uridine tRNA thiolase Ctu1-Ctu2 is required to maintain genome integrity. Proc Natl Acad Sci USA 105: 5459-5464. doi:10.1073/pnas.0709404105

Dilg AWE, Mincione G, Achterhold K, lakovleva O, Mentler M, Luchinat C, Bertini I, Parak FG. 1999. Simultaneous interpretation of Mössbauer, EPR and 57Fe ENDOR spectra of the [Fe4S4] cluster in the high-potential iron protein I from Ectothiorhodospira halophila. J Biol Inorg Chem 4: 727-741. doi:10.1007/s007750050345

Duechler M, Leszczyńska G, Sochacka E, Nawrot B. 2016. Nucleoside modifications in the regulation of gene expression: focus on tRNA. Cell Mol Life Sci 73: 3075-3095. doi:10.1007/s00018-016-2217-y

Emptage MH, Dreyers JL, Kennedy MC, Beinert H. 1983. Optical and EPR characterization of different species of active and inactive aconitase. J Biol Chem 258: 11106-11111.

Flint DH. 1996. Escherichia coli contains a protein that is homologous in function and $\mathrm{N}$-terminal sequence to the protein encoded by the nifS gene of Azotobacter vinelandii and that can participate in the synthesis of the Fe-S cluster of dihydroxy-acid dehydratase. J Biol Chem 271: 16053-16067. doi:10.1074/jbc.271.27.16053

Hagervall TG, Edmonds CG, McCloskey JA, Bjork GR. 1987. Transfer RNA(5-methylaminomethyl-2-thiouridine)-methyltransferase from 
Escherichia coli K-12 has two enzymatic activities. J Biol Chem 262: 8488-8495.

Hagervall TG, Pomerantz SC, McCloskey JA. 1998. Reduced misreading of asparagine codons by Escherichia coli tRNA ${ }^{\text {Lys }}$ with hypomodified derivatives of 5-methylaminomethyl-2-thiouridine in the wobble position. J Mol Biol 284: 33-42. doi:10.1006/jmbi .1998 .2162

Hanson G, Berliner L. 2017. Future directions in metalloprotein and metalloenzyme research. Springer, Heidelberg.

Henshaw TF, Cheek J, Broderick JB. 2000. The [4Fe-4S] ${ }^{1+}$ cluster of pyruvate formate-lyase activating enzyme generates the glycyl radical on pyruvate formate-lyase: EPR-detected single turnover. J Am Chem Soc 122: 8331-8332. doi:10.1021/ja002012q

Hoppe M, Hulthén L, Hallberg L. 2003. Serum iron concentration as a tool to measure relative iron absorption from elemental iron powders in man. Scand J Clin Lab Invest 63: 489-496. doi:10.1080/ 00365510310003003

Hori H, Kawamura T, Awai T, Ochi A, Yamagami R, Tomikawa C, Hirata A. 2018. Transfer RNA modification enzymes from thermophiles and their modified nucleosides in tRNA. Microorganisms 6: E110. doi:10.3390/microorganisms6040110

Horie N, Hara-Yokoyama M, Yokoyama S, Watanabe K, Kuchino Y, Nishimura S, Miyazawa T. 1985. Two tRNAlle1 species from an extreme thermophile, Thermus thermophilus HB8: effect of 2-thiolation of ribothymidine on the thermostability of tRNA. Biochemistry 24: 5711-5715. doi:10.1021/bi00342a004

Ikeuchi Y, Soma A, Ote T, Kato J, Sekine Y, Suzuki T. 2005. Molecular mechanism of lysidine synthesis that determines tRNA identity and codon recognition. Mol Cell 19: 235-246. doi:10.1016/j.molcel 2005.06.007

Ikeuchi Y, Shigi N, Kato J, Nishimura A, Suzuki T. 2006. Mechanistic insights into sulfur relay by multiple sulfur mediators involved in thiouridine biosynthesis at tRNA wobble positions. Mol Cell 21: 97-108. doi:10.1016/j.molcel.2005.11.001

Johansson MJO, Esberg A, Huang B, Bjork GR, Bystrom AS. 2008. Eukaryotic wobble uridine modifications promote a functionally redundant decoding system. Mol Cell Biol 28: 3301-3312. doi:10.1128/MCB.01542-07

Kambampati R, Lauhon CT. 2003. MnmA and IscS are required for in vitro 2-thiouridine biosynthesis in Escherichia coli. Biochemistry 42: 1109-1117. doi:10.1021/bi026536+

Kennedy MC, Mende-Mueller L, Blondin GA, Beinert H. 1992. Purification and characterization of cytosolic aconitase from beef liver and its relationship to the iron-responsive element binding protein. Proc Natl Acad Sci USA 89: 11730-11734. doi:10.1073/ pnas.89.24.11730

Kotera M, Bayashi T, Hattori M, Tokimatsu T, Goto S, Mihara H, Kanehisa M. 2010. Comprehensive genomic analysis of sulfur-relay pathway genes. Genome Inform 24: 104-115. doi:10.1142/ 9781848166585_0009

Kowalak JA, Dalluge JJ, McCloskey JA, Stetter KO. 1994. The role of posttranscriptional modification in stabilization of transfer RNA from hyperthermophiles. Biochemistry 33: 7869-7876. doi:10 .1021/bi00191a014

Larkin MA, Blackshields G, Brown NP, Chenna R, McGettigan PA, McWilliam $H$, Valentin F, Wallace IM, Wilm A, Lopez $R$, et al. 2007. Clustal $W$ and Clustal $X$ version 2.0. Bioinformatics 23: 2947-2948. doi:10.1093/bioinformatics/btm404

Leidel S, Pedrioli PG, Bucher T, Brost R, Costanzo M, Schmidt A, Aebersold R, Boone C, Hofmann K, Peter M. 2009. Ubiquitin-related modifier Urm 1 acts as a sulphur carrier in thiolation of eukaryotic transfer RNA. Nature 458: 228-232. doi:10.1038/nature07643

Letunic I, Bork P. 2016. Interactive tree of life (iTOL) v3: an online tool for the display and annotation of phylogenetic and other trees. Nucleic Acids Res 44: W242-W245. doi:10.1093/nar/gkw290
Liu Y, Zhu X, Nakamura A, Orlando R, Söll D, Whitman WB. 2012. Biosynthesis of 4-thiouridine in tRNA in the methanogenic archaeon Methanococcus maripaludis. J Biol Chem 287: 3668336692. doi:10.1074/jbc.M112.405688

Liu Y, Vinyard DJ, Reesbeck ME, Suzuki T, Manakongtreecheep K, Holland PL, Brudvig GW, Söll D. 2016. A [3Fe-4S] cluster is required for tRNA thiolation in archaea and eukaryotes. Proc Natl Acad Sci USA 113: 12703-12708. doi:10.1073/pnas.1615732113

Milligan JF, Uhlenbeck OC. 1989. Synthesis of small RNAs using T7 RNA polymerase. Methods Enzymol 180: 51-62. doi:10.1016/ 0076-6879(89)80091-6

Morgan TV, Stephens PJ, Devlin F, Burgess BK, Stout CD. 1985. Selective oxidative destruction of iron-sulfur clusters. Ferricyanide oxidation of Azotobacter vinelandii ferredoxin I. FEBS Lett 183: 206-210. doi:10.1016/0014-5793(85)80777-8

Nakagawa H, Kuratani M, Goto-Ito S, Ito T, Katsura K, Terada T, Shirouzu M, Sekine S, Shigi N, Yokoyama S. 2013. Crystallographic and mutational studies on the tRNA thiouridine synthetase TtuA. Proteins 81: 1232-1244. doi:10.1002/prot.24273

Nakai Y, Nakai M, Hayashi H. 2008. Thio-modification of yeast cytosolic tRNA requires a ubiquitin-related system that resembles bacterial sulfur transfer systems. J Biol Chem 283: 27469-27476. doi:10 .1074/jbc.M804043200

Nedialkova DD, Leidel SA. 2015. Optimization of codon translation rates via tRNA modifications maintains proteome integrity. Cell 161: 1606-1618. doi:10.1016/j.cell.2015.05.022

Noma A, Sakaguchi Y, Suzuki T. 2009. Mechanistic characterization of the sulfur-relay system for eukaryotic 2-thiouridine biogenesis at tRNA wobble positions. Nucleic Acids Res 37: 1335-1352. doi:10.1093/nar/gkn1023

Numata T, Ikeuchi Y, Fukai S, Suzuki T, Nureki O. 2006. Snapshots of tRNA sulphuration via an adenylated intermediate. Nature 442: 419-424. doi:10.1038/nature04896

Oashi Z, Saneyoshi M, Harada F, Hara H, Nishimura S. 1970. Presumed anticodon structure of glutamic acid tRNA from E. coli: a possible location of a 2-thiouridine derivative in the first position of the anticodon. Biochem Biophys Res Commun 40: 866-872. doi:10 .1016/0006-291X(70)90983-6

Ranjan N, Rodnina MV. 2017. Thio-modification of tRNA at the wobble position as regulator of the kinetics of decoding and translocation on the ribosome. J Am Chem Soc 139: 5857-5864. doi:10 .1021 /jacs.7b00727

Rodriguez-Hernandez A, Spears JL, Gaston KW, Limbach PA, Gamper H, Hou Y-M, Kaiser R, Agris PF, Perona JJ. 2013. Structural and mechanistic basis for enhanced translational efficiency by 2-thiouridine at the tRNA anticodon wobble position. J Mol Biol 425: 3888-3906. doi:10.1016/j.jmb.2013.05.018

Rozov A, Demeshkina N, Khusainov I, Westhof E, Yusupov M, Yusupova G. 2016. Novel base-pairing interactions at the tRNA wobble position crucial for accurate reading of the genetic code. Nat Commun 7: 10457. doi:10.1038/ncomms10457

Sakaguchi Y, Miyauchi K, Kang B-I, Suzuki T. 2015. Nucleoside analysis by hydrophilic interaction liquid chromatography coupled with mass spectrometry. Methods Enzymol 560: 19-28. doi:10.1016/ bs.mie.2015.03.015

Schaffrath R, Leidel SA. 2017. Wobble uridine modifications-a reason to live, a reason to die?! RNA Biol 14: 1209-1222. doi:10 $.1080 / 15476286.2017 .1295204$

Shigi N. 2014. Biosynthesis and functions of sulfur modifications in tRNA. Front Genet 5: 67. doi:10.3389/fgene.2014.00067

Shigi N. 2018. Recent advances in our understanding of the biosynthesis of sulfur modifications in tRNAs. Front Microbiol 9: 2679. doi:10.3389/fmicb.2018.02679

Shigi N, Sakaguchi Y, Suzuki T, Watanabe K. 2006a. Identification of two tRNA thiolation genes required for cell growth at extremely 
high temperatures. J Biol Chem 281: 14296-14306. doi:10.1074/ jbc.M511675200

Shigi N, Suzuki T, Terada T, Shirouzu M, Yokoyama S, Watanabe K. 2006b. Temperature-dependent biosynthesis of 2-thioribothymidine of Thermus thermophilus tRNA. J Biol Chem 281: 21042113. doi:10.1074/jbc.M510771200

Shigi N, Sakaguchi Y, Asai S, Suzuki T, Watanabe K. 2008. Common thiolation mechanism in the biosynthesis of tRNA thiouridine and sulphur-containing cofactors. EMBO J 27: 3267-3278. doi:10.1038/emboj.2008.246

Shigi N, Asai S-I, Watanabe K. 2016. Identification of a rhodanese-like protein involved in thiouridine biosynthesis in Thermus thermophilus tRNA. FEBS Lett 590: 4628-4637. doi:10.1002/1873-3468 .12499

Stoll S, Schweiger A. 2006. EasySpin, a comprehensive software package for spectral simulation and analysis in EPR. J Magn Reson 178: 42-55. doi:10.1016/j.jmr.2005.08.013

Sweeney WV, Rabinowitz JC, Yoch DC. 1975. High and low reduction potential $4 \mathrm{Fe}-4 \mathrm{~S}$ clusters in Azotobacter vinelandii $\left(4 \mathrm{Fe}-4 \mathrm{~S}^{*}\right)_{2} \mathrm{fer}-$ redoxin I. Influence of the polypeptide on the reduction potentials. J Biol Chem 250: 7842-7847.

Telser J, Lee H-I, Hoffman BM. 2000. Investigation of exchange couplings in [Fe3S4]+ clusters by electron spin-lattice relaxation. J Biol Inorg Chem 5: 369-380. doi:10.1007/PL00010666

Tomari Y, Suzuki T, Watanabe K, Ueda T. 2000. The role of tightly bound ATP in Escherichia coli tRNA nucleotidyltransferase. Genes Cells 5: 689-698. doi:10.1046/j.1365-2443.2000.00360.x

Umeda N, Suzuki T, Yukawa M, Ohya Y, Shindo H, Watanabe K, Suzuki T. 2005. Mitochondria-specific RNA-modifying enzymes responsible for the biosynthesis of the wobble base in mitochondrial tRNAs. Implications for the molecular pathogenesis of human mitochondrial diseases. J Biol Chem 280: 1613-1624. doi:10.1074/ jbc.M409306200

Urbonavicius J, Qian Q, Durand JM, Hagervall TG, Bjork GR. 2001. Improvement of reading frame maintenance is a common function for several tRNA modifications. EMBO J 20: 4863-4873. doi:10 .1093/emboj/20.17.4863

Vendeix FA, Murphy FV, Cantara WA, Leszczyńska G, Gustilo EM, Sproat B, Malkiewicz A, Agris PF. 2012. Human tRNA ${ }^{\text {Lys3 }}$ Uuu is pre-structured by natural modifications for cognate and wobble codon binding through keto-enol tautomerism. J Mol Biol 416: 467-485. doi:10.1016/j.jmb.2011.12.048

Vollmer SJ, Switzer RL, Debrunner PG. 1983. Oxidation-reduction properties of the iron-sulfur cluster in Bacillus subtilis glutamine phosphoribosylpyrophosphate amidotransferase. J Biol Chem 258: 14284-14293.

Watanabe K, Oshima T, Saneyoshi M, Nishimura S. 1974. Replacement of ribothymidine by 5-methyl-2-thiouridine in sequence GT $\psi \mathrm{C}$ in tRNA of an extreme thermophile. FEBS Lett 43: 59-63. doi:10.1016/0014-5793(74)81105-1

Waterhouse A, Bertoni M, Bienert S, Studer G, Tauriello G, Gumienny R, Heer FT, de Beer TAP, Rempfer C, Bordoli L, et al. 2018. SWISS-MODEL: homology modelling of protein structures and complexes. Nucleic Acids Res 46: W296-W303. doi:10 $.1093 / \mathrm{nar} / \mathrm{gky} 427$

Wong JY, Meyer E, Switzer RL. 1977. Glutamine phosphoribosylpyrophosphate amidotransferase from Bacillus subtilis. A novel ironsulfur protein. J Biol Chem 252: 7424-7426.

Yasukawa T, Suzuki T, Ishii N, Ohta S, Watanabe K. 2001. Wobble modification defect in tRNA disturbs codon-anticodon interaction in a mitochondrial disease. EMBO J 20: 4794-4802. doi:10.1093/ emboj/20.17.4794

Yokoyama S, Watanabe T, Murao K, Ishikura H, Yamaizumi Z, Nishimura S, Miyazawa T. 1985. Molecular mechanism of codon recognition by tRNA species with modified uridine in the first position of the anticodon. Proc Natl Acad Sci USA 82: 4905-4909. doi:10.1073/pnas.82.15.4905

Zheng C, Dos Santos PC. 2018. Metallocluster transactions: dynamic protein interactions guide the biosynthesis of $\mathrm{Fe}-\mathrm{S}$ clusters in bacteria. Biochem Soc Trans 46: 1593-1603. doi:10.1042/ BST20180365 

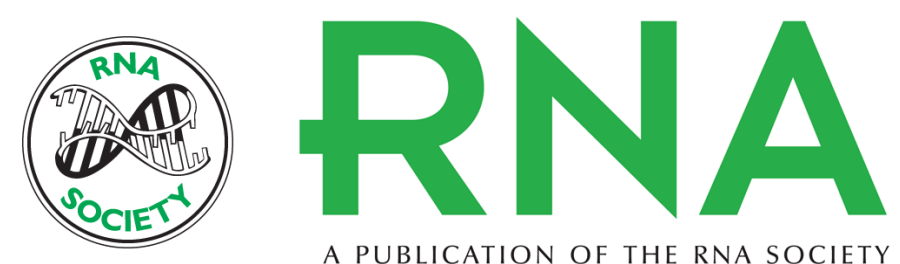

A PUBLICATION OF THE RNA SOCIETY

\section{An ancient type of MnmA protein is an iron-sulfur cluster-dependent sulfurtransferase for tRNA anticodons}

Naoki Shigi, Masaki Horitani, Kenjyo Miyauchi, et al.

RNA 2020 26: 240-250 originally published online December 4, 2019

Access the most recent version at doi:10.1261/rna.072066.119

\section{Supplemental http://rnajournal.cshlp.org/content/suppl/2019/12/04/rna.072066.119.DC1 Material}

References This article cites 73 articles, 27 of which can be accessed free at: http://rnajournal.cshlp.org/content/26/3/240.full.html\#ref-list-1

Creative This article is distributed exclusively by the RNA Society for the first 12 months after the Commons License full-issue publication date (see http://rnajournal.cshlp.org/site/misc/terms.xhtml). After 12 months, it is available under a Creative Commons License (Attribution-NonCommercial 4.0 International), as described at http://creativecommons.org/licenses/by-nc/4.0/.

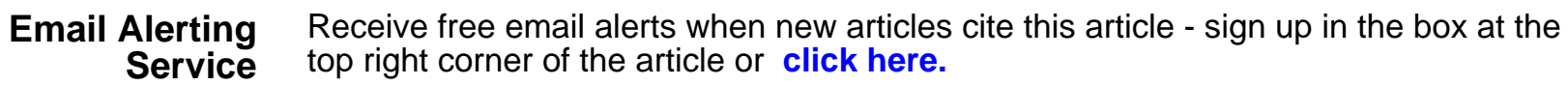

